\title{
Factors Influencing Sleep Difficulty and Sleep Quantity in the Citizen Pscientist Psoriatic Cohort
}

\author{
Mary Patricia Smith $\cdot$ Karen Ly $\cdot$ Quinn Thibodeaux $\cdot$ Thulasi Weerasinghe \\ Kristen Beck - Lindsey Shankle - April W. Armstrong · Marc Boas · Alisha Bridges • \\ Frank Doris · Joel M. Gelfand · Brian Lafoy · Ana-Maria Orbai · Junko Takeshita • \\ Sarah Truman · Marilyn T. Wan · Jashin J. Wu • Michael P. Siegel · Stacie J. Bell • \\ Tina Bhutani $\cdot$ Wilson Liao
}

Received: April 10, 2019 / Published online: June 8, 2019

(C) The Author(s) 2019

\section{ABSTRACT}

Introduction: Sleep is essential for overall health and well-being, yet more than one-third of adults report inadequate sleep. The prevalence is higher among people with psoriasis, with up to $85.4 \%$ of the psoriatic population reporting sleep disruption. Poor sleep among psoriasis patients is particularly concerning because psoriasis is independently associated with many of the same comorbidities as sleep dysfunction, including cardiovascular disease, obesity, and depression. Given the high

Enhanced Digital Features To view enhanced digital features for this article go to https://doi.org/10.6084/ m9.figshare.8208953.

M. P. Smith $(\varangle) \cdot$ K. Ly $\cdot$ Q. Thibodeaux ·

T. Weerasinghe $\cdot$ K. Beck · T. Bhutani - W. Liao Department of Dermatology, University of

California, San Francisco, CA, USA

e-mail: mary.smith2@ucsf.edu

L. Shankle · S. J. Bell

National Psoriasis Foundation, Portland, OR, USA

A. W. Armstrong - M. Boas - A. Bridges - F. Doris · J. M. Gelfand · B. Lafoy · A.-M. Orbai · J. Takeshita S. Truman - M. T. Wan · J. J. Wu - W. Liao Citizen Pscientist Governance Council, National Psoriasis Foundation, Portland, OR, USA

A. W. Armstrong Department of Dermatology, University of Southern California, Los Angeles, CA, USA prevalence and serious consequences of disordered sleep in psoriasis, it is vital to understand the nature of sleep disturbance in this population. This study was designed to help meet this need by using survey data from Citizen Pscientist, an online patient portal developed by the National Psoriasis Foundation.

Methods: Our analysis included 3118 participants who identified as having a diagnosis by a physician of psoriasis alone or psoriasis with psoriatic arthritis. Demographic information, psoriasis severity and duration, sleep apnea status, smoking and alcohol consumption, itch timing, and sleep characteristics were included. Two separate multivariate logistic regression models in STATA were used to determine whether the presence of psoriatic arthritis, age,

J. M. Gelfand · J. Takeshita · M. T. Wan Department of Dermatology, University of Pennsylvania, Philadelphia, PA, USA

A.-M. Orbai

Division of Rheumatology, Johns Hopkins

University School of Medicine, Baltimore, MD, USA

J. J. Wu

Dermatology Research and Education Foundation, Irvine, CA, USA

M. P. Siegel

Pediatric Dermatology Research Alliance, Indianapolis, IN, USA 
gender, body mass index, comorbid sleep apnea, psoriasis severity, timing of worst itch, smoking status, or high-risk alcohol consumption were associated with sleep difficulty or low sleep quantity, defined by the American Academy of Sleep Medicine as less than $7 \mathrm{~h}$ of sleep per night on average.

Results: Results from the multivariate logistic regressions found that sleep difficulty was associated with psoriatic arthritis (OR 2.15, 95\% CI [1.79-2.58]), female gender (2.03 [1.67-2.46]), obese body mass index $(\mathrm{BMI} \geq 30)$ (1.25 [1.00-1.56]), sleep apnea (1.41 [1.07-1.86]), psoriasis severity of moderate (1.59 [1.30-1.94]) or severe (2.40 [1.87-3.08]), and smoking (1.60 [1.26-2.02]). Low sleep quantity was associated with obese BMI (1.62 [1.29-2.03]), sleep apnea (1.30 [1.01-1.68]), psoriasis severity of moderate (1.41 [1.16-1.72]) or severe (1.40 [1.11-1.76]), and smoking (1.62 [1.31-2.00]). Sleep difficulty and low sleep quantity were not associated with age, alcohol consumption, or timing of worst itch.

Conclusion: These results are potentially meaningful in several aspects. We identify an important distinction between sleep difficulty and sleep quantity in psoriatic disease, whereby having psoriatic arthritis and being female are each associated with sleep difficulty despite no association with low sleep quantity. Furthermore, there is conflicting evidence from prior studies as to whether psoriasis severity is associated with sleep difficulty, but this well-powered, large study revealed a strong, graded relationship between psoriasis severity and both sleep difficulty and low sleep quantity. Overall, our results show that both sleep difficulty and low sleep quantity were associated with multiple factors in this analysis of a large psoriatic cohort. These findings suggest that dermatologists may gather clinically useful information by screening psoriatic patients for trouble sleeping and low sleep quantity to identify potential comorbidities and to more effectively guide disease management.

Keywords: Comorbidities; Psoriasis; Sleep; Survey research

\section{INTRODUCTION}

Chronic sleep dysfunction can lead to a wide variety of adverse health outcomes, including cardiovascular disease, hypertension, obesity, type 2 diabetes mellitus, depression, and allcause mortality $[1,2]$. In addition to these longterm consequences, sleep disturbance can profoundly affect daily functioning, contributing to difficulty concentrating, impaired school or work performance, and driving or transportation accidents [3, 4]. Sleep is therefore essential for overall health and well-being [5], yet more than one-third of adults in the USA report inadequate sleep [6]. The prevalence is remarkably higher among people with psoriasis [5, 7-11]. For examples, a 2005 survey found that $49.5 \%$ of psoriasis patients experience sleep interference [7], a 2008 case-control study concluded that disordered sleep is a significant comorbidity in psoriasis [12], and a 2016 systematic review and meta-analysis reported sleep disruption in up to $85.4 \%$ of the psoriatic population [10]. Moreover, people with psoriasis are more likely to have diagnosed sleep-related conditions such as obstructive sleep apnea (OSA) $(36.0-81.8 \%$ prevalence in psoriasis versus $2-4 \%$ in the general population), restless legs syndrome (15.1-18.0\% versus $5-10 \%)$, and transient or chronic insomnia $(5.9-44.8 \%$ versus $10-53 \%$ ) [13].

Poor sleep among psoriasis patients is particularly concerning because psoriasis is independently associated with many of the same comorbidities as sleep dysfunction [12-14]. The consequences of each condition can thereby compound, with deadly results. In fact, a 2016 cohort study found that psoriasis patients with concomitant sleep disorders had significantly higher risks of ischemic heart and stroke events as compared to psoriasis patients without sleep issues [8]. In this way, disordered sleep does not merely reduce quality of life in psoriasis but can result in potentially lethal health outcomes.

Given the high prevalence and serious consequences of disordered sleep in psoriasis, it is vital to better understand the nature of sleep disturbance in this population. Contributing factors can be divided into two categories: 
insufficient quantity of sleep and poor quality of sleep. The former is defined as less $7 \mathrm{~h}$ of sleep per day on average, as recommended by the American Academy of Sleep Medicine [4]. This measure can be readily assessed by directly asking an individual to quantify their average sleep. On the other hand, assessing sleep quality is far more complex. Sleep quality is determined by several factors, including but not limited to sleep latency, continuity, depth, and post-sleep restoration $[15,16]$. An individual may have a subjective sense of their sleep quality and can be screened by asking if they experience difficulty or trouble sleeping on a routine basis, but many require objective evaluation using polysomnography and other specialized methods. Such evaluations are relatively simple and have been studied in association with many medical conditions, but it remains a critical need to more rigorously study sleep disturbance among large numbers of patients living with psoriatic disease [17].

This study was designed to help meet this need. Citizen Pscientist (CP) is an online patient portal developed by the National Psoriasis Foundation (NPF) that is designed to provide psoriatic patients with an opportunity to connect with each other by completing an optional survey with questions including demographic details, symptom and treatment histories, and quality of life topics such as the impact of their condition on diet, exercise, and of course sleep [18]. Participation in CP is completely voluntary. For those who opt in, CP participants can perform their own analyses of responses to the $\mathrm{CP}$ survey since all answers are posted to the $\mathrm{CP}$ website without any identifying information of those who provided it [18]. Participants also have the option to comment on survey results and engage in discussions with other members of the CP community, which has participants around the world [19].

Furthermore, clinicians and researchers can request access to this de-identified data to inform clinical care and research initiatives. The study described here used CP survey data as of July 2018 to examine what factors are associated with sleep difficulty and sleep quantity in this psoriatic cohort.

\section{METHODS}

Our analysis included data from 3118 participants who completed the CP survey and identified as having a diagnosis by a physician of psoriasis alone or psoriasis with psoriatic arthritis. Institutional review board (IRB) approval for CP was obtained from Genetic Alliance, which includes participants outside of the USA as included in this cohort. The study conformed with the Helsinki Declaration of 1964, as revised in 2013, concerning human rights. Participants had access to the Terms and Conditions of $\mathrm{CP}$, including statements regarding the unlikely possibility that a breach of data confidentiality may occur and that their survey responses may be analyzed by researchers studying psoriatic disease and may be presented in written publications [18].

The most recent version of the $\mathrm{CP}$ survey contains 79 questions. A subset of 15 questions were selected for the scope of this analysis (Table 1). Demographic information, psoriasis severity and duration, sleep apnea status, smoking and alcohol consumption, itch timing, and sleep characteristics are provided (Table 2). Psoriasis severity was participant-reported by answering the survey question about psoriasis body surface (BSA) area involvement. Mild was defined as BSA less than 5\%, moderate was defined as BSA of $5-10 \%$, and a BSA greater than $10 \%$ was defined as severe. Sleep characteristics were derived from survey questions about hours of sleep per day on average and trouble sleeping. Participants who reported sleeping less than $7 \mathrm{~h}$ per day on average were categorized as having low sleep quantity, as defined by the American Academy of Sleep Medicine [4], and participants who responded "yes" to the question "Do you have trouble sleeping?" were categorized as having sleep difficulty.

STATA software (version 15) was used to tabulate frequencies, percentages, and means, and two separate multivariate logistic regression models were used to determine whether the presence of psoriatic arthritis, age, gender, body mass index (BMI), comorbid sleep apnea, psoriasis severity, timing of worst itch, smoking status, or high-risk alcohol consumption were 
Table 1 Subset of Citizen Pscientist survey questions and response options

Question Response options

Has a doctor diagnosed you Yes, No

with psoriasis?

Has a doctor diagnosed you Yes, No

with psoriatic arthritis?

How old are you?

What is your sex?

How tall are you?

How much do you weigh?

Do you have one or more of the following conditions?

How much of your body is covered by psoriasis?

At what age did a doctor diagnose you with psoriasis?

At what age did a doctor diagnose you with psoriatic arthritis?

How many cigarettes do you smoke per day?

How many alcoholic drinks do you have per week? (one drink is $12 \mathrm{oz}$ beer, $5 \mathrm{oz}$ wine, $1 \mathrm{oz}$ of liquor)

At what time of day do you experience the most itch?

Do you have trouble sleeping at night?

How many hours of sleep do Under 18, 18-25, 26-30, 31-35, 36-40, 41-45, 46-50, 51-55, 56-60, 61-65, Over 65 Male, Female

Under $4.5 \mathrm{ft}, 4.5 \mathrm{ft}-5 \mathrm{ft}$, Over $5 \mathrm{ft}-5.5 \mathrm{ft}$, Over $5.5 \mathrm{ft}-6 \mathrm{ft}$, Over $6 \mathrm{ft}-6.5 \mathrm{ft}$, Over $6.5 \mathrm{ft}$ Under $100 \mathrm{lbs}, 100-120 \mathrm{lbs}, 121-140 \mathrm{lbs}, 141-160 \mathrm{lbs}, 161-180 \mathrm{lbs}, 181-200 \mathrm{lbs}$, 201-220 lbs, 221-240 lbs, 241-260 lbs, 261-280 lbs, 281-300 lbs, 301-320 lbs, 321-340 lbs, 341-360 lbs, 361-380 lbs, 381-400 lbs, Over $400 \mathrm{lbs}$

Crohn's disease, Juvenile-onset diabetes (type 1), Rheumatoid arthritis, Lupus, Eczema, Cutaneous T cell lymphoma, Celiac disease, Sjogren's syndrome, Uveitis, Thyroid disease, Multiple sclerosis, Alopecia areata, Atopic dermatitis, High cholesterol, High triglycerides, High blood pressure, Coronary artery disease, Stroke, Adult-onset diabetes (type 2), Parkinson's disease, Liver disease, Cancer, Sleep apnea, Fatty liver disease

None or very little (body surface area, BSA $<5 \%$ ), Only a few patches that could be covered by 1 or 2 palms of your hand (BSA $<5 \%$ ), Could be covered by less than 5 palms of your hand (BSA $<5 \%$ ), Between 5 and 10 palms of your hand (BSA 5-10\%), Scattered patches (BSA 5-10\%), Covered by more than 10 palms of your hand $($ BSA $>10 \%)$, Extensive psoriasis (BSA > 10\%)

I have never been diagnosed with psoriasis by a doctor, Under 18, 18-25, 26-30, 31-35, 36-40, 41-45, 46-50, 51-55, 56-60, 61-65, Over 65

I have never been diagnosed with psoriatic arthritis by a doctor, Under 18, 18-25, $26-30,31-35,36-40,41-45,46-50,51-55,56-60,61-65$, Over 65

I don't smoke, I only smoke occasionally, 1-10 cigarettes, 11-20 cigarettes, More than 20 cigarettes

I don't drink alcohol, 1-3, 4-6, 7-9, 10 or more

Morning, Midday, Afternoon, Evening, Night

Yes, No

Under $5 \mathrm{~h}, 5 \mathrm{~h}, 6 \mathrm{~h}, 7 \mathrm{~h}, 8 \mathrm{~h}, 9 \mathrm{~h}, 10$ or more hours you get per day on average? 
associated with sleep difficulty or low sleep quantity (Table 3). The dependent variables listed above were chosen a priori, and both models were evaluated and adjusted for statistical interactions. For each outcome, $p$ values of less than 0.05 were deemed significant, and odds ratios (ORs) with 95\% confidence intervals (CIs) are reported.

\section{RESULTS}

Demographic results and clinical characteristics (Table 2) demonstrate that the CP population is almost equally composed of participants with psoriasis only ( $n=1588,50.9 \%)$ and those with psoriatic arthritis $(n=1530,49.1 \%)$. As previously described, the CP psoriatic cohort is approximately 70\% White/Caucasian [19]. This population is predominantly female $(n=2153$, $69.1 \%$ ), and the mean age is 45.2 (standard deviation 0.3). The average BMI is 27.6 (standard deviation 0.3 ), and $62.8 \%$ of participants have an overweight or obese BMI. With respect to disease duration, more than 10 years was reported by the majority of both psoriasis $(80.3 \%)$ and psoriatic arthritis (69.5\%) participants. In terms of sleep characteristics, over half of participants reported sleep difficulty (58.4\%), and low sleep quantity was reported by $38.8 \%$ of respondents. More specifically, the proportions of participants endorsing each survey response option for sleep duration are illustrated in Fig. 1.

Results from the multivariate logistic regressions (Table 3) found that sleep difficulty was associated with psoriatic arthritis (OR 2.15, 95\% CI [1.79-2.58]), female gender (2.03 $[1.67-2.46])$, obese body mass index (BMI $\geq 30)$ (1.25 [1.00-1.56]), sleep apnea (1.41 [1.07-1.86]), psoriasis severity of moderate (1.59 [1.30-1.94]) or severe $(2.40[1.87-3.08])$, and smoking (1.60 [1.26-2.02]). Low sleep quantity was associated with obese BMI (1.62 [1.29-2.03]), sleep apnea (1.30 [1.01-1.68]), psoriasis severity of moderate (1.41 [1.16-1.72]) or severe (1.40 [1.11-1.76]), and smoking (1.62 [1.31-2.00]). Sleep difficulty and low sleep quantity were not associated with age, alcohol consumption, or timing of worst itch.

\section{DISCUSSION}

These results are potentially meaningful in several aspects. First, we identify an important distinction between sleep difficulty and sleep quantity in psoriatic disease, whereby having psoriatic arthritis and being female are each associated with sleep difficulty despite no association with low sleep quantity. It is not possible to definitively account for these differences from this analysis, but various potential explanations can be considered. It is feasible that psoriatic arthritis contributes to musculoskeletal discomfort that causes more sleep difficulty than the cutaneous symptoms of psoriasis alone. Such physical discomfort might not reduce the number of hours slept on average but may cause trouble initiating and staying asleep or feeling rested after a sufficient quantity of sleep [7], which may be reported by CP participants as sleep difficulty despite adequate sleep quantity. With respect to gender, an extensive literature review indicates that this is the first study to report that female gender is uniquely associated with sleep difficulty in psoriatic patients. It is conceivable that women with psoriasis are more likely to report trouble sleeping because they tend to be more attuned to less restorative sleep, as reported in the general population [20]. Thus, even though women with psoriasis report similar quantity of sleep as their male counterparts, the former might not be sleeping as soundly as the latter who also may be less aware of any problem.

Second, there is conflicting evidence from prior studies as to whether psoriasis severity is associated with sleep difficulty $[7,9]$, but this is a well-powered, large study that revealed a strong, graded relationship between psoriasis severity and both sleep difficulty and low sleep quantity. These associations are reasonable given that more severe physical symptoms of psoriasis, especially pruritus [21], are more disruptive to a person's ability to sleep without difficulty, delayed onset, or premature cessation. Likewise, a 2008 study found that impaired sleep quality can partly mediate the association between pruritus and psychological symptoms in psoriasis, including anxiety and 
Table 2 Clinical and sleep characteristics

\begin{tabular}{|c|c|}
\hline Characteristic & Frequency $(\%)$ \\
\hline \multicolumn{2}{|l|}{ Disease } \\
\hline Psoriasis & $1588(50.9)$ \\
\hline Psoriasis with psoriatic arthritis & $1530(49.1)$ \\
\hline \multicolumn{2}{|l|}{ Age } \\
\hline $18-20$ & $142(4.6)$ \\
\hline $21-30$ & $248(8.0)$ \\
\hline $31-40$ & $559(17.9)$ \\
\hline $41-50$ & $696(22.3)$ \\
\hline $51-60$ & $806(25.8)$ \\
\hline $61-70$ & $653(20.9)$ \\
\hline $71-80$ & $8(0.3)$ \\
\hline $81-90$ & $5(0.2)$ \\
\hline $91-99$ & $1(0.1)$ \\
\hline \multicolumn{2}{|l|}{ Gender } \\
\hline Male & $960(30.8)$ \\
\hline Female & $2153(69.1)$ \\
\hline No response & $5(0.1)$ \\
\hline \multicolumn{2}{|l|}{ Body mass index (BMI) } \\
\hline Underweight $(<18.5)$ & $137(4.4)$ \\
\hline Normal (18.5-24.9) & $1021(32.8)$ \\
\hline Overweight (25-29.9) & $875(28.1)$ \\
\hline Obese $(\geq 30)$ & $1085(34.8)$ \\
\hline \multicolumn{2}{|l|}{ Comorbid sleep apnea } \\
\hline Absent & $2735(87.7)$ \\
\hline Present & $383(12.3)$ \\
\hline \multicolumn{2}{|l|}{ Psoriasis severity } \\
\hline Mild ( $<5 \%$ body surface area) & $1328(45.1)$ \\
\hline Moderate (5-10\% body surface area) & $1053(35.8)$ \\
\hline Severe $(>10 \%$ body surface area $)$ & $564(19.2)$ \\
\hline \multicolumn{2}{|l|}{ Disease duration } \\
\hline \multicolumn{2}{|l|}{ Psoriatic skin disease } \\
\hline $0-10$ years & $613(19.7)$ \\
\hline$>10$ years & $2505(80.3)$ \\
\hline
\end{tabular}

Table 2 continued

\begin{tabular}{lc}
\hline Characteristic & Frequency (\%) \\
\hline Psoriatic arthritis & \\
$0-10$ years & $466(30.5)$ \\
$>10$ years & $1064(69.5)$ \\
Smoking status & \\
Non-smoker & $2416(80.3)$ \\
Smoker & $594(19.7)$ \\
Alcohol consumption & \\
Low-risk & $2710(89.7)$ \\
High-risk & $311(10.3)$ \\
\hline Timing of Worst Itch & Frequency (\%) \\
\hline Do not notice a difference & $202(6.5)$ \\
Morning & $363(11.6)$ \\
Midday & $1704(58.4)$ \\
Afternoon & $307(9.8)$ \\
Evening & $349(11.1)$ \\
Night & $1048(33.7)$ \\
\hline Sleep characteristics & \\
\hline Noep quality & \\
\hline
\end{tabular}

depression [22]. Such psychological comorbidities are more common in moderate and severe psoriasis and also independently associated with sleep disturbance [11, 23-26]. Therefore, sleep problems in psoriasis may be due to a combination of disease severity, as seen in this study, as well as closely related psychological factors that undoubtedly play a role in these associations but that were outside the scope of the CP survey. 
Table 3 Factors associated with sleep difficulty or low sleep quantity by multivariate logistic regression

\begin{tabular}{|c|c|c|c|}
\hline Variable & OR & $95 \% \mathrm{CI}$ & $p$ value \\
\hline \multicolumn{4}{|l|}{ Sleep difficulty ${ }^{a}$} \\
\hline \multicolumn{4}{|l|}{ Disease } \\
\hline Psoriasis without psoriatic arthritis & - & - & - \\
\hline Psoriasis with psoriatic arthritis & 2.15 & $1.79-2.58$ & $<0.001^{*}$ \\
\hline Age & 0.99 & $0.93-1.04$ & 0.671 \\
\hline \multicolumn{4}{|l|}{ Gender } \\
\hline Male & - & - & - \\
\hline Female & 2.03 & $1.67-2.46$ & $<0.001^{*}$ \\
\hline \multicolumn{4}{|l|}{ BMI } \\
\hline Normal BMI & - & - & - \\
\hline Underweight $(<18.5)$ & 0.81 & $0.53-1.24$ & 0.331 \\
\hline Overweight (25-29.9) & 1.08 & $0.86-1.36$ & 0.493 \\
\hline Obese $(\geq 30)$ & 1.25 & $1.00-1.56$ & $0.047^{*}$ \\
\hline Comorbid sleep apnea & 1.41 & $1.07-1.86$ & $0.016^{*}$ \\
\hline \multicolumn{4}{|l|}{ Psoriasis severity } \\
\hline Mild & - & - & - \\
\hline Moderate & 1.59 & $1.30-1.94$ & $<0.001^{*}$ \\
\hline Severe & 2.40 & $1.87-3.08$ & $<0.001^{*}$ \\
\hline \multicolumn{4}{|l|}{ Timing of worst itch } \\
\hline No difference & - & - & - \\
\hline Morning & 1.26 & $0.62-2.56$ & 0.521 \\
\hline Midday & 1.19 & $0.58-2.43$ & 0.641 \\
\hline Afternoon & 1.30 & $0.64-2.66$ & 0.468 \\
\hline Evening & 1.18 & $0.60-2.33$ & 0.630 \\
\hline Night & 1.34 & $0.68-2.65$ & 0.403 \\
\hline Smoker & 1.60 & $1.26-2.02$ & $<0.001^{*}$ \\
\hline High-risk alcohol consumption & 0.79 & $0.59-1.05$ & 0.105 \\
\hline \multicolumn{4}{|l|}{ Sleep quantity ${ }^{\mathrm{b}}$} \\
\hline \multicolumn{4}{|l|}{ Disease } \\
\hline Psoriasis without psoriatic arthritis & - & - & - \\
\hline Psoriasis with psoriatic arthritis & 1.08 & $0.91-1.29$ & 0.371 \\
\hline Age & 1.01 & $0.96-1.07$ & 0.663 \\
\hline Gender & & & \\
\hline
\end{tabular}


Table 3 continued

\begin{tabular}{|c|c|c|c|}
\hline Variable & OR & $95 \% \mathrm{CI}$ & $p$ value \\
\hline Male & - & - & - \\
\hline Female & 1.16 & $0.95-1.40$ & 0.138 \\
\hline \multicolumn{4}{|l|}{ BMI } \\
\hline Normal BMI & - & - & - \\
\hline Underweight $(<18.5)$ & 1.15 & $0.75-1.76$ & 0.527 \\
\hline Overweight (25-29.9) & 1.09 & $0.86-1.38$ & 0.475 \\
\hline Obese $(\geq 30)$ & 1.62 & $1.29-2.03$ & $<0.001^{*}$ \\
\hline Comorbid sleep apnea & 1.30 & $1.01-1.68$ & $0.044^{*}$ \\
\hline \multicolumn{4}{|l|}{ Psoriasis severity } \\
\hline Mild & - & - & - \\
\hline Moderate & 1.41 & $1.16-1.72$ & $0.001^{*}$ \\
\hline Severe & 1.40 & $1.11-1.76$ & $0.004^{*}$ \\
\hline \multicolumn{4}{|l|}{ Timing of worst itch } \\
\hline No difference & - & - & - \\
\hline Morning & 0.81 & $0.40-1.63$ & 0.553 \\
\hline Midday & 0.96 & $0.47-1.93$ & 0.900 \\
\hline Afternoon & 0.91 & $0.45-1.84$ & 0.797 \\
\hline Evening & 0.98 & $0.50-1.90$ & 0.943 \\
\hline Night & 0.84 & $0.43-1.65$ & 0.618 \\
\hline Smoker & 1.62 & $1.31-2.00$ & $<0.001^{*}$ \\
\hline High-risk alcohol consumption & 1.34 & $0.70-2.58$ & 0.372 \\
\hline
\end{tabular}

$O R$ odds ratio, $C I$ confidence interval

${ }^{*} p$ value of less than 0.05 was deemed to be significant

a Defined as answering "yes" to the survey question "Do you have trouble sleeping at night?"

b Defined as answering "Under $5 \mathrm{~h}$, " $5 \mathrm{~h}$," or " $6 \mathrm{~h}$ " to the survey question "How many hours of sleep do you get per day on average?"

- Indicates the reference group for each variable

Third, it is important to discuss the influence of OSA status and treatment on these results. Comorbid OSA was found to be associated with both sleep difficulty and low sleep quantity. These findings are consistent with literature on the relationship between OSA and psoriasis $[9,13,27,28]$. At the same time, the CP survey did not include questions about treatment for OSA, such as continuous positive airway pressure (CPAP), that could certainly influence the relationships between sleep difficulty, sleep quantity, and psoriasis. Similarly, high BMI was associated with both sleep variables, but given the association between being overweight or obese with OSA and psoriasis [29, 30], the link between high BMI and poor sleep may be explained by undiagnosed OSA. Relatedly, prior studies with smaller psoriatic cohorts have not 


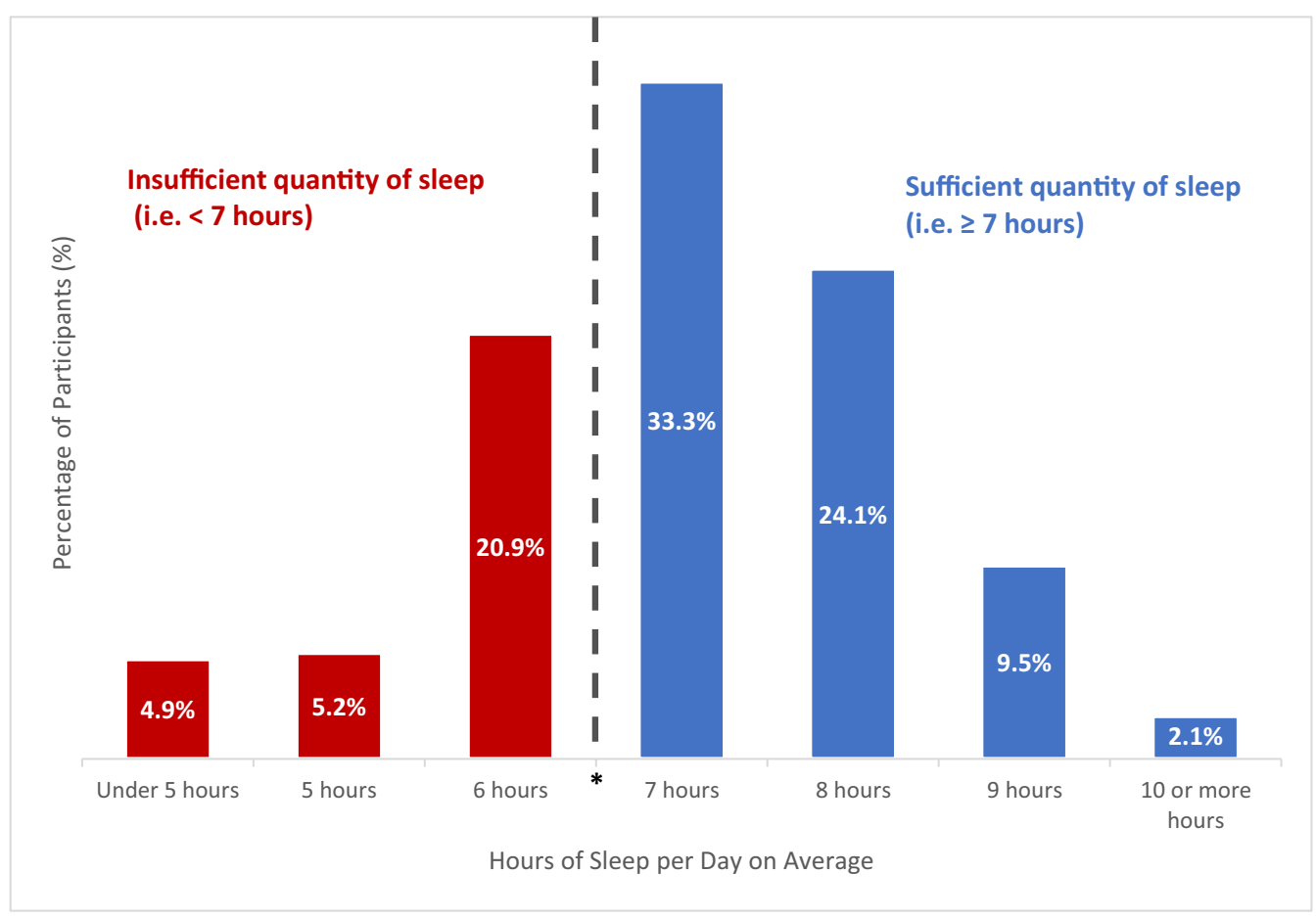

Fig. 1 Sleep duration responses. *American Academy of Sleep Medicine recommends $7 \mathrm{~h}$ as the amount of sleep needed to promote optimal health in adults [4]

found obesity to be predictive of sleep interference [7], but this analysis demonstrated a strong relationship between high BMIs and both sleep difficulty and low sleep quantity.

\section{Limitations}

All data used for this analysis were participantreported and not physician-confirmed. For example, psoriasis severity was determined by patient-reported evaluation of BSA involvement rather than a physician's evaluation. Another example is that OSA status was reported by patients who may or may not have been formally evaluated for this key sleep-related comorbidity. This limitation could not be overcome given the web-based delivery of the CP survey and the de-identification of survey responses, making it not possible to link $\mathrm{CP}$ participant responses to physician-reported data.

Another limitation is that causality cannot be inferred from the reported associations. This is a limitation inherent to data from observational cohort studies at a single time point like this one. A related limitation is that the design of this study as an online survey for patients living with psoriatic disease makes it not possible to compare the results from participants' responses to a control group.

An additional limitation is related to the survey instrument. As discussed, the full CP survey contains 79 questions that span many topics related to living with psoriatic disease. Only two of these questions relate to sleep difficulty and quantity. While both questions were included in the subset used for this analysis (Table 1), it would have been helpful to have more questions about sleep, such as asking about non-restorative sleep, sleep timing, or many other factors that influence sleep quality and duration. It would have been additionally helpful to include questions about psychological symptoms, such as anxiety or depression that could also directly impact sleep as described above. Using a more extensive, validated 
instrument to evaluate sleep in psoriatic patients would be appropriate for future studies.

Lastly, the CP participant population lacks racial, gender, and age diversity. With particular respect to race, this cohort is approximately $70 \%$ White/Caucasian. Although psoriasis is most common in this group [31], a more racially diverse cohort would better capture a broader range of experiences with psoriasis and sleep.

\section{CONCLUSIONS}

Overall, our results show that both sleep difficulty and low sleep quantity were associated with multiple factors in this analysis of a large psoriatic cohort. These findings suggest that dermatologists may gather clinically useful information by screening psoriatic patients for trouble sleeping and low sleep quantity to identify potential comorbidities and to more effectively guide disease management. For example, if a patient endorses trouble sleeping or low sleep quantity, the physician could consider comorbidities such as obesity, OSA, and psychological symptoms. In some cases, a referral to a sleep specialist may be necessary for a more thorough evaluation of the many nuanced factors that influence sleep [16]. Incorporating such screening into routine dermatologic care seems not only manageable but also essential given the well-established high prevalence of sleep dysfunction in psoriasis as well as the severe health outcomes associated with this comorbidity in the psoriatic population $[1,5,7-11,13,21]$.

Moreover, several studies have examined whether treatment of psoriasis alone is associated with improvements in sleep disturbance among this population. Results have shown that sleep problems can significantly improve with successful treatment of psoriasis, leading to reduced daytime fatigue and improved quality of life [32, 33]. However, sleep dysfunction may not remit entirely even when other psoriatic symptoms are well controlled, especially if there are sleep-related comorbidities such as OSA [34]. These findings reinforce the importance of screening psoriatic patients for sleep problems and suggest that it may not be sufficient to only treat psoriasis in patients who endorse sleep disturbance.

In conclusion, this analysis helps fill the critical need to better understand the many associations of sleep disturbance in psoriasis and derives from an innovative approach to patient-centered research, whereby the psoriatic population is directly involved in the design, conduct, and analysis of the results [19]. While this study has several limitations as discussed, a key strength is overcoming selection bias that can occur in sleep research since CP participants were not selected on this basis, but rather identified through a select subset of CP survey answers. It is important to continue pioneering and advocating for research like this that make patients feel empowered and supported while also giving researchers and clinicians greater insight into many aspects of their lives with psoriatic disease, including the impact on sleep that has been thus far inadequately explored given its many consequences.

\section{ACKNOWLEDGEMENTS}

We are grateful to the many participants who have contributed to the $\mathrm{CP}$ initiative. We are thankful for the CP participants who participated in study design and implementation. These participants continue to contribute valuable feedback and improvements to $\mathrm{CP}$ as active members of our CP governing council.

Funding. The development of Citizen Pscientist (CP) was funded by the National Psoriasis Foundation and partially funded through a Patient-Centered Outcomes Research Institute (PCORI) Pipeline-to-Proposal Award, administered on behalf of PCORI by the Colorado Foundation for Public Health and the Environment. No funding was received for article processing charges.

Authorship. All named authors meet the International Committee of Medical Journal Editors (ICMJE) criteria for authorship for this article, take responsibility for the integrity of 
the work as a whole, and have given their approval for this version to be published.

Prior Presentation. No component of this manuscript has been posted, submitted, published, or presented elsewhere. The figures and tables are all original and have been produced by the authors for this particular publication.

Disclosures. Mary Patricia Smith, Karen Ly, Quinn Thibodeaux, Lindsey Shankle, Thulasi Weerasinghe, Kristen Beck, Marilyn T. Wan, Frank Doris, Alisha Bridges, Marc Boas, Brian Lafoy, Michael P. Siegel and Sarah Truman have nothing to disclose. The National Psoriasis Foundation receives unrestricted financial support from AbbVie, Inc., Amgen, Inc., Celgene Corporation, Eli Lilly and Company, Janssen Biotech, Inc., LEO Pharma Inc., Mallinckrodt Pharmaceuticals, Novartis Pharmaceuticals, Pfizer, Inc. and Valeant Pharmaceuticals International, Inc. Stacie J. Bell is employed by the National Psoriasis Foundation. April W. Armstrong has served as investigator, advisor, and/ or consultant to AbbVie, Janssen, Novartis, Eli Lilly, Regeneron Pharmaceuticals, Inc., Sanofi U.S., and Ortho Dermatologics, Inc. Joel M. Gelfand served as a consultant for Bristol-Myers Squibb, Boehringer Ingelheim, GlaxoSmithKline, Janssen Biologics, Novartis, Union Chimique Belge (UCB), Sanofi and Pfizer Inc., receiving honoraria; and receives research grants (to the Trustees of the University of Pennsylvania) from Abbvie, Janssen, Novartis, Celgene, Ortho Dermatologics, and Pfizer Inc. and received payment for continuing medical education work related to psoriasis that was supported indirectly by Lilly, Ortho Dermatologics, Inc. and Novartis. Dr. Gelfand is a copatent holder of resiquimod for treatment of cutaneous $\mathrm{T}$ cell lymphoma. Dr Gelfand is a Deputy Editor for the Journal of Investigative Dermatology, receiving honoraria from the Society for Investigative Dermatology. Junko Takeshita also receives a research grant from Pfizer (to the Trustees of the University of Pennsylvania) and has received payment for continuing medical education work related to psoriasis that was supported indirectly by Eli Lilly. Ana-Maria Orbai is funded in part by grants from the National Institutes of Health (P30 AR070254-01), the Rheumatology Research Foundation (RRF) Scientist Development Award, and the Staurulakis Discovery Award. Ana-Maria Orbai has served as an investigator and received research funding (to Johns Hopkins University) from Abbvie, Celgene, Janssen, Horizon, Eli Lilly and Novartis. In addition, A.M.O. has also served as a consultant for Eli Lilly, Janssen, Novartis, Pfizer and Union Chimique Belge (UCB). Jashin J. Wu is an investigator for AbbVie, Amgen, Eli Lilly, Janssen and Novartis and a consultant for AbbVie, Almirall, S.A., Amgen, Bristol-Myers Squibb, Celgene, Dermira, Dr. Reddy's Laboratories, Eli Lilly, Janssen, LEO Pharma, Novartis, Promius Pharma LLC, Regeneron, Sun Pharmaceutical Industries Ltd., Union Chimique Belge (UCB) and Valeant Pharmaceuticals North America LLC; and is a speaker for AbbVie, Celgene, Novartis, Regeneron, Sun Pharmaceutical, Union Chimique Belge (UCB) and Valeant. Tina Bhutani has received research funding from the National Psoriasis Foundation and has served as a research investigator and/or consultant for Eli Lilly, Janssen, Merck, Celgene and Regeneron. Wilson Liao is funded in part by grants from the National Institutes of Health (R01AR065174, U01AI119125) and has served as a research investigator for Abbvie, Amgen, Janssen, Novartis, Pfizer and Regeneron.

Compliance with Ethics Guidelines. IRB approval for CP was obtained from Genetic Alliance, which includes participants outside of the USA. The study conformed with the Helsinki Declaration of 1964, as revised in 2013, concerning human and animal rights, and that Springer's policy concerning informed consent has been followed.

Data Availability. The data sets generated and/or analyzed during the current study are available from the National Psoriasis Foundation on reasonable request. Please contact the corresponding author for more information.

Open Access. This article is distributed under the terms of the Creative Commons Attribution-NonCommercial 4.0 International 
License (http://creativecommons.org/licenses/ by-nc/4.0/), which permits any noncommercial use, distribution, and reproduction in any medium, provided you give appropriate credit to the original author(s) and the source, provide a link to the Creative Commons license, and indicate if changes were made.

\section{REFERENCES}

1. Itani O, Jike M, Watanabe N, Kaneita Y. Short sleep duration and health outcomes: a systematic review, meta-analysis, and meta-regression. Sleep Med. 2017;32:246-56.

2. Malik SW, Kaplan J. Sleep deprivation. Prim Care. 2005;32(2):475-90.

3. Centers for Disease Control and Prevention (CDC). Effect of short sleep duration on daily activities-United States, 2005-2008. MMWR Morb Mortal Wkly Rep. 2011;60(8):239-42.

4. Watson NF, Badr MS, Belenky G, et al. Recommended amount of sleep for a healthy adult: a joint consensus statement of the American Academy of Sleep Medicine and Sleep Research Society. Sleep. 2015;38(6):843-4.

5. Jensen P, Zachariae C, Skov L, Zachariae R. Sleep disturbance in psoriasis: a case-controlled study. $\mathrm{Br}$ J Dermatol. 2018;179(6):1376-84.

6. Liu Y, Wheaton AG, Chapman DP, Cunningham TJ, $\mathrm{Lu} \mathrm{H}$, Croft JB. Prevalence of healthy sleep duration among adults-United States, 2014. MMWR Morb Mortal Wkly Rep. 2016;65(6):137-41.

7. Callis Duffin K, Wong B, Horn EJ, Krueger GG. Psoriatic arthritis is a strong predictor of sleep interference in patients with psoriasis. J Am Acad Dermatol. 2009;60(4):604-8.

8. Chiu HY, Hsieh CF, Chiang YT, et al. Concomitant sleep disorders significantly increase the risk of cardiovascular disease in patients with psoriasis. PLoS One. 2016;11(1):e0146462.

9. Henry AL, Kyle SD, Chisholm A, Griffiths CEM, Bundy C. A cross-sectional survey of the nature and correlates of sleep disturbance in people with psoriasis. Br J Dermatol. 2017;177(4):1052-9.

10. Henry AL, Kyle SD, Bhandari S, Chisholm A, Griffiths CE, Bundy C. Measurement, classification and evaluation of sleep disturbance in psoriasis: a systematic review. PLoS One. 2016;11(6):e0157843.
11. Wong ITY, Chandran V, Li S, Gladman DD. Sleep disturbance in psoriatic disease: prevalence and associated factors. J Rheumatol. 2017;44(9):1369-74.

12. Wu Y, Mills D, Bala M. Psoriasis: cardiovascular risk factors and other disease comorbidities. J Drugs Dermatol. 2008;7(4):373-7.

13. Gupta MA, Simpson FC, Gupta AK. Psoriasis and sleep disorders: a systematic review. Sleep Med Rev. 2016;29:63-75.

14. Shah K, Mellars L, Changolkar A, Feldman SR. Realworld burden of comorbidities in US patients with psoriasis. J Am Acad Dermatol. 2017;77(2):287-92.e4.

15. Martin SE, Wraith PK, Deary IJ, Douglas NJ. The effect of nonvisible sleep fragmentation on daytime function. Am J Respir Crit Care Med. 1997;155(5):1596-601.

16. Harvey AG, Stinson K, Whitaker KL, Moskovitz D, Virk H. The subjective meaning of sleep quality: a comparison of individuals with and without insomnia. Sleep. 2008;31(3):383-93.

17. Henry AL, Kyle SD, Bundy C. A call for improved sleep research in psoriasis populations. Int J Dermatol. 2016;55(5):e312.

18. National Psoriasis Foundation. Citizen pscientist terms and conditions. 2018. https://pscientist. psoriasis.org/terms. Accessed 4 May 2019.

19. Sanchez IM, Shankle L, Wan MT, et al. Building a citizen pscientist: advancing patient-centered psoriasis research by empowering patients as contributors and analysts. Dermatol Ther (Heidelb). 2018;8(3):405-23.

20. Boccabella A, Malouf J. How do sleep-related health problems affect functional status according to sex? J Clin Sleep Med. 2017;13(5):685-92.

21. Kaaz K, Szepietowski JC, Matusiak L. Influence of itch and pain on sleep quality in atopic dermatitis and psoriasis. Acta Derm Venereol. 2019;99(2):175-80.

22. Zachariae R, Zachariae CO, Lei U, Pedersen AF. Affective and sensory dimensions of pruritus severity: associations with psychological symptoms and quality of life in psoriasis patients. Acta Derm Venereol. 2008;88(2):121-7.

23. Shutty BG, West C, Huang KE, et al. Sleep disturbances in psoriasis. Dermatol Online J. 2013;19(1):1.

24. Gowda S, Goldblum OM, McCall WV, Feldman SR. Factors affecting sleep quality in patients with psoriasis. J Am Acad Dermatol. 2010;63(1):114-23. 
25. Ohayon MM, Roth T. Place of chronic insomnia in the course of depressive and anxiety disorders. J Psychiatr Res. 2003;37(1):9-15.

26. Nicholas MN, Gooderham M. Psoriasis, depression, and suicidality. Skin Therapy Lett. 2017;22(3):1-4.

27. Shalom G, Dreiher J, Cohen A. Psoriasis and obstructive sleep apnea. Int J Dermatol. 2016;55(11):e579-84.

28. Hirotsu C, Nogueira H, Albuquerque RG, Tomimori J, Tufik S, Andersen ML. The bidirectional interactions between psoriasis and obstructive sleep apnea. Int J Dermatol. 2015;54(12):1352-8.

29. Kabeloglu Ilbay V, Tas B, Altuntas M, Atakli HD, Soysal A. Risk of obstructive sleep apnea syndrome in psoriasis patients. Arch Iran Med. 2019;22(3):137-43.

30. Malhotra A, White DP. Obstructive sleep apnoea. Lancet. 2002;360(9328):237-45.
31. Rachakonda TD, Schupp CW, Armstrong AW. Psoriasis prevalence among adults in the United States. J Am Acad Dermatol. 2014;70(3):512-6.

32. Thaci D, Galimberti R, Amaya-Guerra $M$, et al. Improvement in aspects of sleep with etanercept and optional adjunctive topical therapy in patients with moderate-to-severe psoriasis: results from the PRISTINE trial. J Eur Acad Dermatol Venereol. 2014;28(7):900-6.

33. Strober BE, Sobell JM, Duffin KC, et al. Sleep quality and other patient-reported outcomes improve after patients with psoriasis with suboptimal response to other systemic therapies are switched to adalimumab: results from PROGRESS, an open-label phase IIIB trial. Br J Dermatol. 2012;167(6):1374-81.

34. Maari C, Bolduc C, Nigen S, Marchessault P, Bissonnette R. Effect of adalimumab on sleep parameters in patients with psoriasis and obstructive sleep apnea: a randomized controlled trial. J Dermatolog Treat. 2014;25(1):57-60. 